\title{
Efficacy of mupirocin nasal ointment in eradicating Staphylococcus aureus nasal carriage in chronic haemodialysis patients
}

\author{
D. L. Holton', L. E. Nicolle ${ }^{2}$, D. Diley ${ }^{3}$ and K. Bernstein ${ }^{4}$ \\ ${ }^{1,2}$ Sections of Infectious Diseases and ${ }^{3,4}$ Nephrology, ${ }^{1,2}$ Department of Medical \\ Microbiology and ${ }^{3,4}$ Department of Medicine, University of Manitoba
}

Accepted for publication 22 October 1990

\begin{abstract}
Summary: Topical $2 \%$ mupirocin ointment eradicated chronic Staphylococcus aureus nasal carriage immediately post-therapy in $17(77 \%)$ of 22 haemodialysis patients. Mean time to recurrence was 3.8 weeks. Similar pretherapy and post-therapy phage types occurred in $12(71 \%)$ of 17 patients. Staphylococcus aureus infections developed in none of 17 successfully treated patients, two of five treatment failures $(P=0.05)$, and 10 of 46 untreated patients studied concurrently $(P=0.03)$.
\end{abstract}

Keywords: Mupirocin; haemodialysis; Staphylococcus aureus; nasal colonization.

\section{Introduction}

Infection is the second leading cause of morbidity and mortality in haemodialysis patients. Staphylococcus aureus is the most significant pathogen, accounting for $70-96 \%$ of bacterial infections. ${ }^{1-3}$ In a prospective study, $\mathrm{Yu}$ et al. $^{4}$ showed a decreased incidence of $S$. aureus infections if $S$. aureus nasal carriage was eliminated. Until recently, only systemic therapy with either rifampicin or clindamycin reliably eradicated $S$. aureus nasal carriage in haemodialysis patients. ${ }^{5}$ These drugs are expensive and may be associated with significant toxicity. Topical gentamicin, vancomycin, bacitracin and oral cloxacillin or tetracycline have interrupted $S$. aureus nasal carriage for less than 2 weeks before relapse occurred. ${ }^{5}$

Mupirocin is a new topical antimicrobial which has been reported to effectively eradicate $S$. aureus nasal carriage in some patient populations ${ }^{6,7}$ including haemodialysis patients. ${ }^{8}$ Mupirocin reversibly binds to bacterial isoleucyl-transfer RNA synthetase preventing protein and RNA

Correspondence to: Dr D. Holton, Health and Welfare Canada, Laboratory Center for Disease Control, Tunney's Pasture, Ottawa, Ontario, Canada KIA 0L2. 
synthesis.,10 It is active against most Gram-positive skin pathogens, although low and high level resistance has been reported. ${ }^{11-13}$

We undertook this pilot study to determine: (a) the efficacy of mupirocin in eliminating nasal carriage in a dialysis population and (b) the length of time patients remained free of nasal $S$. aureus carriage following treatment.

\section{Methods}

\section{Population and design}

All patients attending an outpatient chronic haemodialysis unit were eligible for enrolment. Sixty-eight patients were screened for the presence of $S$. aureus nasal carriage. Patients with two consecutive nasal swabs, taken at least one week apart, positive for $S$. aureus, were considered to be chronic $S$. aureus carriers and enrolled after obtaining informed consent. No subject was known to be hypersensitive to mupirocin.

Patients applied $2 \%$ mupirocin ointment in a paraffin/lanolin base three times daily for 5 days to the internal, anterior medial septa (Little's area) of both nostrils following directions provided by the study nurse. Each patient recorded drug administration and adverse reactions. The study nurse reviewed patient diaries and discussed any adverse drug reactions with each patient. Surveillance of the haemodialysis unit for S. aureus nasal carriage was discontinued after study enrolment was completed. A concurrent prospective study documented infectious morbidity and mortality of all patients in the unit throughout the surveillance and study period.

\section{Microbiology}

Nasal swabs for $S$. aureus carriage were taken at pre-enrolment, at the first dialysis visit following completion of therapy (end of therapy), weekly for 4 weeks, then monthly for 2 months. Specimens were transported to the laboratory in Amies transport media, inoculated onto 5\% sheep blood agar and $10 \%$ mannitol salt agar plates, and incubated aerobically at $37^{\circ} \mathrm{C}$ for 48 hours. Gram-positive coagulase positive cocci isolated from the blood agar plate were identified as $S$. aureus. Oxacillin disc ( $1 \mathrm{mg}, \mathrm{BDL})$ sensitivities were performed on all $S$. aureus isolates. Isolates growing within $13 \mathrm{~mm}$ of the oxacillin disc were inoculated onto Mueller Hinton plates containing $6 \mathrm{mg} \mathrm{l}^{-1}$ oxacillin to verify methicillin resistance. Mupirocin susceptibility was determined by Kirby-Bauer agar disc method $(1 \mu \mathrm{g}$ disc). Staphylococcus aureus isolates were phage typed by the Laboratory Center for Disease Control, Ottawa, Canada.

\section{Definitions and data analysis}

Patients were considered cured if $S$. aureus was not isolated in any post-therapy cultures. If $S$. aureus was isolated on the first nasal swab obtained after completion of therapy, patients were designated failures. Patients relapsed when the $S$. aureus strain isolated post-therapy did not 
differ by more than two phage types from the pre-therapy isolate and were considered recolonized if the post therapy strain differed by more than two phage types. Patients with non-typable strains isolated both pre-therapy and post-therapy were considered relapses. Analysis was performed using the $\chi^{2}$-square test.

This phase two trial was approved by the University of Manitoba, Faculty Committee on Human Subjects in Research and the Health Sciences Centre Research Committee.

\section{Results}

Twenty-two $(32 \%)$ of 68 paticnts in the haemodialysis unit were identified as $S$. aureus nasal carriers during the 70-day surveillance study which preceded the mupirocin trial. All 22 patients were enrolled in the trial. Twelve different phage types were identified in these 22 patients. Seventeen $(77 \%)$ patients were culture negative at the end of therapy. The mean time to recurrence for those negative at the end of therapy was 3.8 weeks. Ten $(46 \%)$ patients remained culture negative 4 weeks post-therapy, seven $(32 \%)$ at 2 months, and five $(23 \%)$ at 3 months. The positive nasal carriers at 3 months included five $(23 \%$ of total) failures of therapy, seven $(32 \%)$ relapses and five $(23 \%)$ recolonized. Thus, similar pre- and post-therapy phage types occurred in 12 of $17(71 \%)$ patients not cured. All pre- and post-therapy $S$. aureus isolates were susceptible to mupirocin. The single subject with a pre-treatment methicillin-resistant $S$. aureus isolated was a treatment failure.

Therapy was well tolerated. Three $(13 \%)$ of 22 patients complained of mild nasal itching. Although one of these patients missed three doses, the course of therapy was subsequently completed. An additional patient missed four doses for unknown reasons. Staphylococcus aureus nasal carriage recurred in both of these patients, one as a relapse, and one recolonized.

None of the 17 patients who were culture negative at completion of therapy developed $S$. aureus infections during the three months follow-up. Staphylococcus aureus cellulitis was observed, however, in two $(40 \%)$ of five patients who failed therapy $(P=0.05)$. Ten $(22 \%)$ of 46 patients not enrolled in the study but followed concurrently had $S$. aureus infection $(P=0.03)$. These 10 infections included three fistula site infections, three episodes of sepsis, two pneumonias, and one each of cellulitis and soft tissue infection.

\section{Discussion}

These observations contrast with the report of Chow $\& \mathrm{Yu}^{5}$ who found less than $25 \%$ of patients using topical agents such as gentamicin, vancomycin or bacitracin remained culture negative 3-14 days after completing treatment. Our results are, however, similar to the observed response to 
systemic therapy. $\mathrm{Yu}$ et $a l^{4}$ found $75 \%$ of patients remained culture negative after combined therapy with oral rifampicin and topical bacitracin 1 month after therapy, but most patients had positive nasal cultures for $S$. aureus by 3 months after therapy. Boelaert et al. ${ }^{8}$ have also recently reported that continuously administered mupirocin eradicated $S$. aureus nasal carriage in haemodialysis patients and was associated with a decreased incidence of $S$. aureus infections. Although they reported no mupirocin resistance, the continual administration of drugs would likely increase the potential for resistance to develop. Our study suggests that the intermittent use of mupirocin may also be effective in eradicating $S$. aureus carriage in haemodialysis and is associated with a significant decrease in $S$. aureus infections.

We observed a higher relapse rate and lower recolonization rate than was observed in a study using a 5-day course of mupirocin to eradicate chronic $S$. aureus nasal carriage in a group of 32 healthy subjects. Casewell \& Hill ${ }^{6}$ found $10(71 \%)$ of 14 patients recolonized and four $(29 \%)$ relapsed during 22 weeks of follow-up. These authors postulated that patients who relapsed were colonized with $S$. aureus at other sites. This hypothesis may explain why our patients had a higher rate of relapse. Compared to healthy controls, haemodialysis patients have twice the rate of $S$. aureus skin colonization. ${ }^{14-16}$ Staphylococcus aureus skin colonization has recently been correlated with personal hygiene in haemodialysis patients. ${ }^{17}$ As this study did not include any evaluation of personal hygiene, it is not known whether the patients who failed had poorer personal hygiene. The level of personal hygiene may be relevant to the two patients who developed $S$. aureus infections after failing to eliminate $S$. aureus nasal carriage.

This preliminary information suggests that a prospective, randomized placebo-controlled trial to determine efficacy and cost benefit of mupirocin in this population is warranted.

This work was supported by a grant from Beecham Laboratory, Canada. We wish to acknowledge the technical assistance of Dr Shirley Parker, Donna Collins, Debbie Godal, Val Weselowski, Assunta Rendina and Lori Takada. Secretarial assistance was provided by Cheryl Reimer and Brenda Kowalczyk.

\section{References}

1. Lundin AP, Adler AJ, Feinroth MV, Berlyne GM, Friedman EA. Maintenance hemodialysis: survival beyond the first decade. $7 A M A$ 1980; 244: 38-40.

2. Neff MS, Eiser AR, Baez A, Gupta S, Amarga E. Patients surviving ten years of hemodialysis. Am Y Med 1983; 74: 996-1004.

3. Bradley JR, Evan DB, Calne RV. Long-term survival in haemodialysis patients. Lancet 1987; 1: 295-296.

4. Yu VL, Goetz A, Wagner M, Smith PB, Rihs JD, Hanchett J, Zuravleff JJ. Staphylococcus aureus nasal carriage and infection in patients on hemodialysis: efficacy of antibiotic prophylaxis. $N$ Engl $\mathcal{F}$ Med 1986; 315: 91-96.

5. Chow JW, Yu VL. Staphylococcus aureus nasal carriage in hemodialysis patients: its role in infection and approaches to prophylaxis. Arch Intern Med 1989; 149: 1258-1262. 
6. Casewell MW, Hill R. Elimination of nasal carriage of Staphylococcus aureus with mupirocin (pseudomonic acid)--a controlled trial. $\mathscr{f}$ Antimicrob Chemother 1986; 17: 365-372.

7. Hill R, Duckworth GJ, Casewell W. Elimination of nasal carriage of methicillinresistant Staphylococcus aureus with mupirocin during a hospital outbreak. F Antimicrob Chemother 1988; 22: 377-384.

8. Boelaert J, De Smedt RA, De Baere YA et al. The influence of calcium mupirocin nasal ointment on the incidence of Staphylococcus aureus infections in haemodialysis patients, Nephrol Dial Transplant 1989; 4: 278-281.

9. Ward A, Campoli-Richards DM. Mupirocin-a review of its antibacterial activity, pharmacokinetic properties and therapeutic use. Drugs 1986; 32: 425-444.

10. Parenti MA, Hatfield SM, Leyden JJ. Mupirocin: a topical antibiotic with a unique structure and mechanism of action. Clin Pharm 1987; 6: 761-770.

11. Kavi J, Andrews JM, Wise R. Mupirocin-tesistant Staphylococcus aureus, Lancet 1987; 2: $1472-1473$.

12. Rahman M, Noble WC, Cookson B. Mupirocin-resistant Staphylococcus aureus, Lancet. 1987; 2: 387--388.

13. Smith GE, Kennedy CTC. Staphylococcus aureus resistance to mupirocin. F Antimicrob Chemother 1988; 21: 141-142.

14. Goldblum SE, Ulrich JA, Goldman RS, Reed WP. Nasal and cutaneous flora among hemodialysis patients and personnel: quantitative and qualitative characterization and patients of Staphylococcus carriage. Amer $\mathcal{F}$ Kidney Dis 1982; 2: 281--286.

15. Wheat LJ, Kohler RB, White AL, White A. Effect of rifampin on nasal carriers of coagulase-positive staphylococci. I Infect Dis 1981; 144: 177.

16. Berman DS, Schaefler S, Simberkoff MS, Rahal JJ. Staphylococcus aureus colonization in intravenous drug users, dialysis patients, and diabetics. F Infect Dis 1987; 155: 829-831.

17. Kaplowitz LG, Comstock JA, Landwehr DM, Dalton HP, Mayhall CG. Prospective study of microbial colonization of the nose and skin and infection of the vascular access site in hemodialysis patients. F Clin Micrabiol 1988; 2: 1257-1262. 